\title{
Enseñanza-Aprendizaje En La Estancia Clínica: Percepción Desde Los Estudiantes De Licenciatura En Enfermería
}

\author{
Eliazar González Carrillo, Dra. \\ Norma Pizarro, Dra. \\ Oscar Joel Talavera Sánchez, Dr. \\ Salvador Vargas García, ME \\ Universidad Autónoma de Chihuahua, México
}

doi: 10.19044/esj.2017.v13n16p313 URL:http://dx.doi.org/10.19044/esj.2017.v13n16p313

\begin{abstract}
The clinical teaching of nursing graduates is one of the fundamental pillars for the development of knowledge, skills, attitudes, and values that would be projected in the real scenarios of their disciplinary practice. The expectation of any institution in professional formation in health discipline is that the facilitator should create an environment that is favorable for an effective teaching-learning process to take place. During the stay of the students in the healthcare institution, it is essential that they acquire a prospective, sensitive, and a specialized vision about their practice and the challenges they face in an increasingly complex society. There are a number of contradictions between theory and practice in care. This, however, leads to a clear gap which becomes evident in traditional teaching models. This requires a redirection towards innovative, humanistic, and specialized approaches to more dynamic ones in this information/knowledge society. The objective of this study was to obtain the perception of undergraduate nursing students on clinical teaching and whether it is related to the hospital context, experience, and teaching profile. Therefore, this favors an effective learning process. It is a quantitative, non-experimental, analytical, descriptive, cross-sectional, and prospective study with a convenience sample of 212 students in a sixth, seventh, and eighth semester of the clinical stationed (practice) class. Among the most important results of the research, it was found that the students stated that there is no functional relationship between Jean Watson's Human Care Theory and its application in the clinical field. They also perceived that this learning had a significant impact on several factors both of the Clinical environment and the educational institution. The study concludes that the students' perception of the teaching-
\end{abstract}


learning process oscillates between the contradiction of a theory that cannot be projected in practice and a series of factors that have a negative impact on the formation of their professional competences.

Keywords: Learning, clinical stay, perception

\section{Resumen}

La enseñanza clínica de los licenciados en enfermería es uno de los pilares fundamentales para el desarrollo de conocimientos, habilidades, actitudes y valores que habrán de proyectar en los escenarios reales de su práctica disciplinar. La expectativa de toda institución formadora de profesionales en el área de la salud es que el facilitador cree el entorno y las condiciones favorables para que suceda un proceso efectivo de enseñanzaaprendizaje. Durante el tiempo que permanecen los estudiantes en la institución asistencial es imprescindible que adquieran una visión prospectiva, sensible y especializada sobre su praxis y los retos que enfrentan en una sociedad cada vez más compleja. Existe una serie de contradicciones entre la teoría y la práctica en el cuidado que evidencia una clara ruptura con los modelos de enseñanza tradicionales y exige una redirección hacia los enfoques innovadores, humanistas y especializados de la dinámica en la sociedad del conocimiento. El objetivo de este estudio es analizar la percepción que tiene el estudiante de licenciatura en enfermería sobre la enseñanza clínica y si esta se relaciona con el contexto hospitalario, experiencia y perfil docente, favoreciendo así un proceso efectivo de aprendizaje, en la Facultad de Enfermería de la Universidad Autónoma de Chihuahua. Es un estudio con enfoque cuantitativo, con diseño no experimental, analítico, descriptivo, trasversal, prospectivo, con un muestreo a conveniencia de 212 estudiantes en estancia clínica de sexto, séptimo y octavo semestre. Entre los resultados más importantes de la investigación se encontró que los estudiantes consideran que no existe una relación funcional entre la Teoría del Cuidado Humano de Jean Watson y su aplicación en el campo clínico, así mismo perciben que su aprendizaje es se impactado por varios factores tanto del ambiente clínico como de la institución educativa. El estudio concluye que la percepción de los estudiantes sobre el proceso de enseñanza-aprendizaje oscila entre la contradicción de una teoría que no alcanza a proyectarse en la práctica y una serie de factores que impactan negativamente en la formación de sus competencias profesionales.

Palabras Clave: Aprendizaje, estancia clínica, percepción 


\section{Introducción}

Entidades como la Organización Panamericana de Salud (OPS), la Organización Mundial de la Salud (OMS) y los Comités Interinstitucionales para la Evaluación de la Educación Superior (CIESS) han trazado las políticas en materia educativa que actualmente definen la gestión académica.

La Universidad Autónoma de Chihuahua, no puede deslindarse de este perfil con visión humanista, técnico y especializado que ha impuesto el nuevo contexto sociocultural en el paradigma moderno y ha entrado en esta fase formativa de profesionales de la salud comprometidos con su entorno.

El actual modelo educativo de la UACH busca, a través de sus ejes los cuales son: centrado en el aprendizaje basado en competencias y en un currículo flexible, responder a los retos que surgen en la dinámica social con una visión globalizada y sensible ante los cambios que suceden en los entornos internacionales.

En esa construcción de los perfiles profesionales, se plantea como fundamento para la práctica cotidiana de la enfermería, la Teoría del Cuidado Humano de la Dra. Jean Watson.

Desde esta perspectiva, el eje de análisis de la investigación busca conocer y analizar la percepción de los estudiantes en las estancias clínicas sobre su proceso de enseñanza-aprendizaje, una tarea que resulta esencial para redireccionar las acciones formativas y evaluar la funcionalidad del modelo o plan curricular vigente.

Pinto (2002) señala que la enfermería es una disciplina que cumple dos tareas primordiales: el cuidado de los pacientes y la enseñanza. Las enfermeras que se dedican a la docencia - a través de un compromiso ético y moral acorde a las necesidades que demandan las instituciones de salud y la sociedad - deben realizar esfuerzos destacados para promover el aprendizaje en los estudiantes que cursan sus prácticas clínicas.

El aprendizaje se debe evaluar mediante un proceso objetivo que mida de forma precisa el progreso formativo y la adquisición de competencias en la práctica clínica, en este sentido, resulta imprescindible examinar la percepción de los estudiante para redirigir las acciones institucionales y potenciar la calidad de la enseñanza.

Crespo (2009) define algunas de las características de la práctica profesional en enfermería como: un conjunto de conocimientos disciplinares, la aplicación del marco ético legal de la profesión, la sensibilidad para ofrecer una atención humanista, la integración con el equipo multidisciplinario, ser un gestor del cuidado y tener la habilidad para la toma de decisiones en diferentes contextos.

Bernal-Becerril y Ponce-Gómez (2009) describen en su Propuesta para la Enseñanza del Cuidado en Enfermería, que la teoría disciplinar del 
cuidado ha sido edificada a través de una compleja metodología participativa y en constante construcción.

La enseñanza en enfermería -dicen- se ha construido a través de dos niveles de actuación: uno como formadores profesionales y otro como agente cuidador del individuo, grupo o familias. Las investigadoras resaltan la importancia de la teoría del Aprendizaje Significativo de David Ausubel y su impacto en la práctica educativa al considerar las experiencias previas del estudiante y un enfoque más constructivo del conocimiento.

Es interesante encontrar estudios con enfoques cuantitativos relacionados con la percepción del alumno de enfermería sobre el desempeño docente en la práctica clínica. La mayoría de estos proyectos académicos coinciden en que existen diferencias sustanciales entre la teoría y la práctica por lo que se cree conveniente revisar, analizar y comparar los resultados con la presente investigación.

Dentro de los estudios en el área de la salud, Palacios-Gutiérrez y Quiroga-Lagos (2012) realizaron un estudio para evaluar a estudiantes de Odontología en relación a las características y comportamientos del profesor asociados a una enseñanza clínica efectiva.

Entre los resultados de la investigación se destacaron fortalezas como “respeto por los estudiantes” y “destrezas comunicacionales”, debilidades en las dimensiones de “organización” así como "disponibilidad y disposición para ayudar”.

En términos comparativos, las investigaciones describen las áreas de oportunidad o mejora en la enseñanza clínica, conocer otros escenarios en la dinámica del cuidado permite reconocer las líneas de acción institucionales y evaluar la funcionalidad de sus proyectos.

La dinámica funcional del proceso enseñanza-aprendizaje implica proveer en los estudiantes los conocimientos, habilidades actitudes y valores a través del comportamiento del profesor clínico, una proyección implica no sólo las bases teóricas sino la construcción de un entorno flexible para que suceda con éxito un cambio duradero de conducta como resultado de la experiencia vivida en escenarios reales ideas (Reyes, Mendoza \& Barrera, 2015).

En otro estudio realizado en dos universidades privadas de Bogotá, Colombia, por Mantilla-Pastrana y Gutiérrez-Agudelo (2013) y denominado "Procesos de evaluación del aprendizaje del cuidado en la práctica de estudiantes de enfermería”, los resultados fueron muy similares en relación al bajo número de competencias y objetivos de aprendizaje relacionados explícitamente con el conocimiento del cuidado de enfermería.

De acuerdo a este estudio, no existe un modelo efectivo para evaluar la enseñanza del cuidado en la práctica de la enfermería que oriente los procesos de aprendizaje y los retroalimente. En este sentido, rescatar la 
percepción de los estudiantes en el proceso enseñanza-aprendizaje dentro de la práctica clínica es un factor indispensable para redefinir las líneas curriculares de los modelos educativos.

Moreno-Tello, Prado-Moncivais y García-Avendaño (2013) realizaron un estudio sobre los ambientes de aprendizaje dentro de la práctica clínica y consideraron una nueva forma de organizar la enseñanza acorde al paradigma socioeducativo moderno.

Esta alternativa, en relación, al modelo tradicional implicaba el empleo de la tecnología y la creación de un entorno educativo centrado en el estudiante para fomentar su autoaprendizaje, el desarrollo del pensamiento crítico y una visión innovadora y prospectiva de su práctica profesional.

De acuerdo a los autores, para que suceda con éxito la enseñanza se requiere establecer un ambiente de aprendizaje clínico propicio, acorde con las metas institucionales, con reglas de comportamiento aceptadas por los estudiantes y estrategias áulicas diseñadas de acuerdo a su estado de desarrollo cognitivo, social y moral.

De acuerdo a un estudio realizado por Cáneo (2016), la percepción del ambiente educacional y su entorno de aprendizaje es un aspecto muy importante en la educación de las Ciencias de la Salud. Esta visión objetiva de los estudiantes sobre su propio proceso de conocimiento se relaciona con altos niveles de satisfacción y logros académicos durante su carrera, ¿̇la razón?: es el análisis crítico de los factores que inciden en su éxito estudiantil.

Desde el punto de vista académico existen muchas interrogantes sobre la funcionalidad de la enseñanza y las estrategias efectivas en la construcción del conocimiento. Investigar la percepción de los estudiantes sobre su proceso de aprendizaje en el ambiente clínico es una tarea fundamental para tomar decisiones que permitan mejorar la calidad de la enseñanza.

La práctica clínica es uno de los pilares del aprendizaje que resulta fundamental en la formación de profesionales del cuidado en enfermería, en la actualidad representa un reto ineludible para el docente lograr que sus estudiantes incorporen en sus estancias clínicas un modelo de cuidado sensible y en una dimensión técnica muy eficaz que cumpla con las expectativas del paciente.

Además, conocer la percepción que tienen los estudiantes del proceso enseñanza-aprendizaje en las estancias clínicas permite identificar fortalezas y debilidades y con ello generar propuestas para mejorar los aspectos que inciden en su formación profesional.

En este sentido, el objetivo de este estudio, es determinar la percepción que tiene el estudiante de enfermería sobre la enseñanza en la estancia clínica y si esta se relaciona con la experiencia y el perfil docente 
para lograr el aprendizaje. La investigación se realizó en la Facultad de Enfermería y Nutriología de la Universidad Autónoma de Chihuahua en el periodo comprendido de marzo - diciembre del año 2015.

Existe la necesidad apremiante de rescatar -a través de investigaciones científicas- la visión de los estudiantes de la licenciatura en enfermería acerca del vínculo entre la teoría y la práctica en los escenarios reales, dentro de las instituciones prestadoras de servicios de salud que actúan como campos clínicos y conocer si el docente ha facilitado estos aprendizajes (Betancourt, et al., 2011).

La evaluación representa un procedimiento intencionado, funcional, continuo e integral, cuyo objetivo final es obtener información sobre diversos aspectos de los fenómenos educativos, especialmente valorar la pertinencia de los programas y el desempeño de los profesores en la estancia clínica y el aprovechamiento de los estudiantes, es una acción ineludible en el proceso formativo de los profesionales en el área de la salud.

\section{Metodología}

La presente investigación es un estudio descriptivo, de corte transversal, se analizaron los datos con un enfoque cuantitativo en una población de 212 estudiantes del $6^{\circ}, 7^{\circ}$ y $8^{\circ}$ semestre de la Licenciatura en Enfermería de la Universidad Autónoma de Chihuahua.

Los escenarios fueron instituciones de primero y segundo nivel de atención, el tiempo de la recolección de datos se realizó cuando los estudiantes se encontraban realizando sus prácticas clínicas, de acuerdo al plan de estudios 2005 aún vigente.

Se elaboró instrumento estructurado tipo Escala de Likert para la recolección de los datos contempla un apartado para datos generales y otros seis para dimensiones: Relación Teoría -Práctica, Aplicación de la Teoría de Jean Watson en la Estancia Clínica, Aprendizaje en la Estancia Clínica, Competencia Docente en la Estancia Clínica, Habilidades Docentes para la Enseñanza Clínica, Evaluación del Aprendizaje y Ambiente Hospitalario.

El instrumento se diseño con un total de 96 ítems y fue adaptado del cuestionario sobre La percepción de los alumnos de enfermería y los factores que inciden en su aprendizaje clínico de la Universidad Nacional Autónoma de México, Facultad de Estudios Superiores Zaragoza (CrespoKnopfler, González-Velázquez \& Agama-Sarabia, 2011).

Se aplicó una prueba piloto con 33 estudiantes que se encontraban en prácticas y se validó el instrumento mediante la prueba de Alfa de Cronbach, con un resultado de 0.95 de confiabilidad. 


\begin{tabular}{|c|c|c|c|c|c|c|c|}
\hline & \multicolumn{7}{|c|}{ DIMENSIONES } \\
\hline & $\begin{array}{c}\text { Relación } \\
\text { Teoría - } \\
\text { Práctica. }\end{array}$ & $\begin{array}{c}\text { Aplicación } \\
\text { de Teoría } \\
\text { Watson }\end{array}$ & $\begin{array}{l}\text { Aprendizaje } \\
\text { en Estancia } \\
\text { Clínica }\end{array}$ & $\begin{array}{l}\text { Aspectos } \\
\text { Docentes }\end{array}$ & $\begin{array}{l}\text { Evaluación } \\
\text { de los } \\
\text { Aprendizajes }\end{array}$ & $\begin{array}{l}\text { Ambiente } \\
\text { Hospitalario }\end{array}$ & Todos \\
\hline $\begin{array}{l}\text { Alfa de } \\
\text { Conbrach }\end{array}$ & 0.885 & 0.823 & 0.810 & 0.986 & 0.918 & 0.737 & 0.971 \\
\hline $\begin{array}{l}\text { Número de } \\
\text { elementos }\end{array}$ & 10 & 10 & 12 & 34 & 11 & 10 & 87 \\
\hline Casos válidos & 211 & 211 & 211 & 211 & 211 & 211 & 211 \\
\hline Casos excluidos & 0 & 0 & 0 & 0 & 0 & 0 & 0 \\
\hline Total & 211 & 211 & 211 & 211 & 211 & 211 & 211 \\
\hline
\end{tabular}

Fuente Directa

El rigor científico de la investigación radica en los criterios éticos (principio de beneficencia y respeto a la dignidad humana). y de audibilidad (credibilidad, confiabilidad, através del consentimiento informado, audibilidad y transferibilidad).

\section{Resultados y discusión}

La muestra de esta investigación se creó a conveniencia con un total de 212 estudiantes que realizaban sus prácticas clínicas en instituciones del sector salud en los turnos matutino, vespertino, nocturno y jornada acumulada.

El instrumento se diseñó para identificar datos socio demográficos en seis dimensiones: Teoría-Práctica, Aplicación de la Teoría de Jean Watson, Aprendizaje Estancia Clínica, Competencia Docente, Evaluación de aprendizaje y Ambiente Hospitalario, los resultados más relevantes se describen en las siguientes figuras:

Figura 1. Percepción de los estudiantes en relación a la enseñanza-aprendizaje en la estancia clínica de la Licenciatura en Enfermería respecto al género en el Ciclo Escolar Enero-Junio 2015.

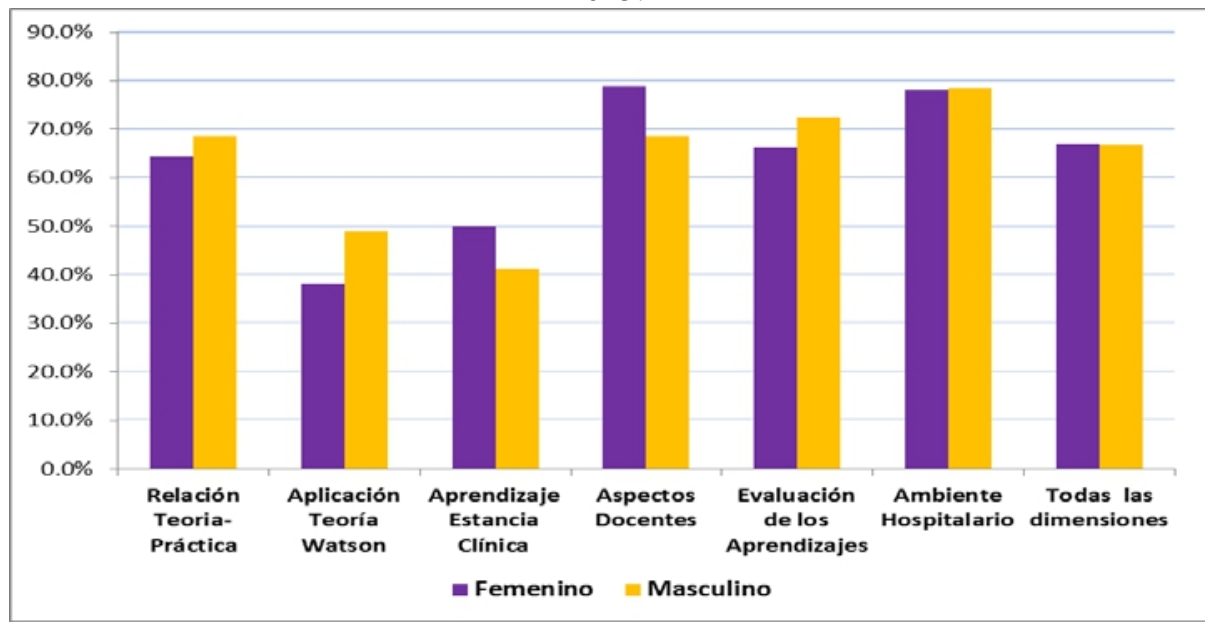

Fuente directa. 
La Figura 1 muestra resultados positivos en la dimensión teoríapráctica, competencia docente, evaluación del aprendizaje y ambiente hospitalario, sin embargo, en la aplicación de la teoría de Jean Watson y el aprendizaje en la estancia clínica -dos de los aspectos más relevantes en la formación profesional de los enfermería- los resultados son negativos (38-49 $\%$ y 50 y $41 \%$ respectivamente)

Los resultados coinciden con un estudio de Sanhueza y Zavala (2007) donde evaluaron el aprendizaje de los estudiantes a partir de sus experiencias en el cuidado de las personas. Esta investigación le daba más importancia a la participación activa de los estudiantes, privilegiando el pensamiento crítico, reflexivo y analítico para resolver problemas y entender mejor la realidad y dinámica de su profesión.

Figura 2. Percepción de los estudiantes en relación a la enseñanza-aprendizaje en la estancia clínica de la Licenciatura en Enfermería respecto a la formación docente en la Teoría del

Cuidado Humano de Jean Watson en el Ciclo Escolar Enero-Junio 2015.

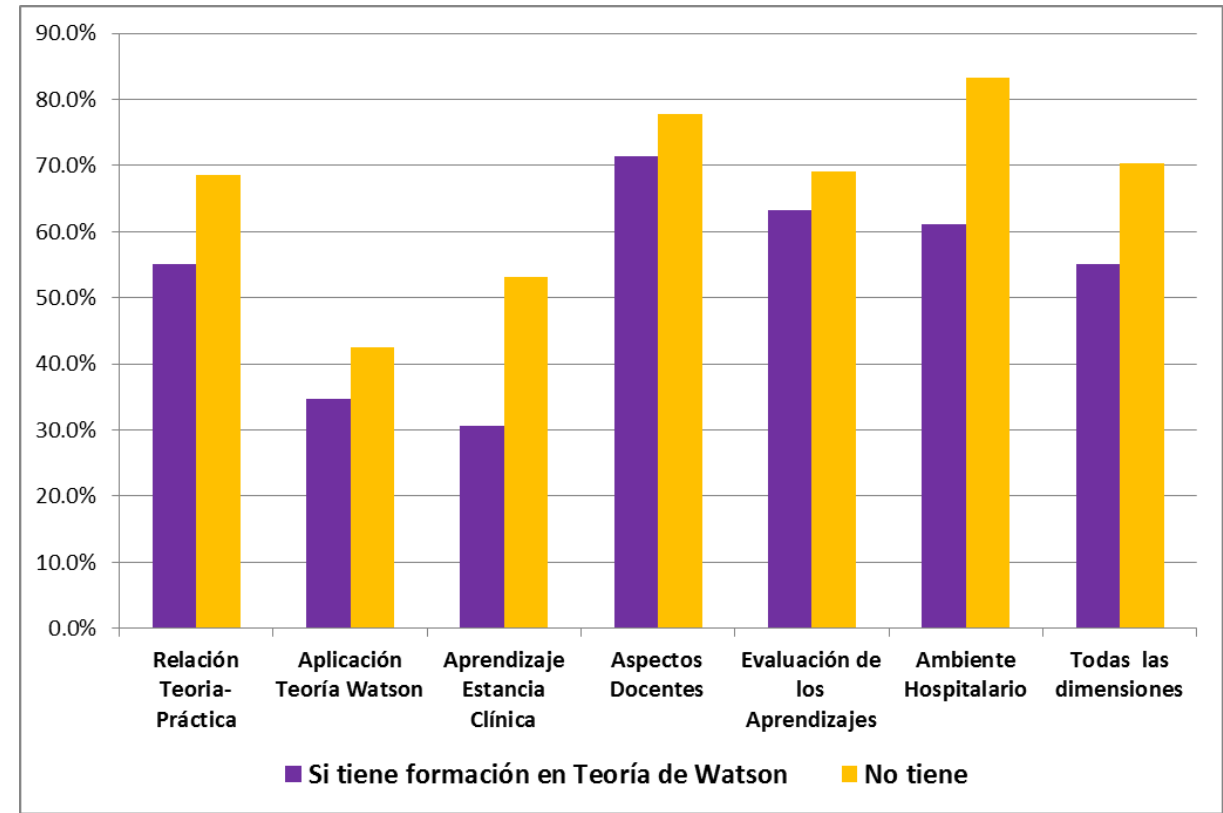

Fuente directa.

La teoría de Jean Watson es un constructo técnico-filosófico que intenta rescatar el cuidado desde la perspectiva humanística y siempre preservando la dignidad de la persona. En el impulso de la modernidad, la tecnología, la apertura de los mercados mundiales y el acceso a las redes de comunicación, el cuidado a la persona se ha visto fragmentado, tornándose rutinario y despersonalizado. El currículo de la licenciada en enfermería son en su totalidad mujeres, se sustenta en ésta teoría y dentro de su contenido se sitúan algunas unidades de aprendizaje específicas para su desarrollo. Sin embargo, en el transcurso de la investigación se evidencian resultados que 
contradicen todo enfoque teórico del ideario de Jean Watson en su aplicación en el contexto hospitalario y las competencias docentes.

Gasull (2005), destaca aspectos positivos en la vinculación que existe entre las estructuras académicas y asistenciales. Las enfermeras enfatizan que si la filosofía del cuidado que se practica en el hospital se debe a las competencias adquiridas en las instituciones educativas entonces la teoría de Jean Watson no han permeado funcionalmente hacia los centros de salud e incluso los docentes sólo se han quedado en un plano teórico sin lograr transmitirla al área clínica.

Figura 3. Percepción de los estudiantes en relación a la enseñanza-aprendizaje en la estancia clínica de la Licenciatura en Enfermería respecto al semestre en el Ciclo Escolar EneroJunio 2015.

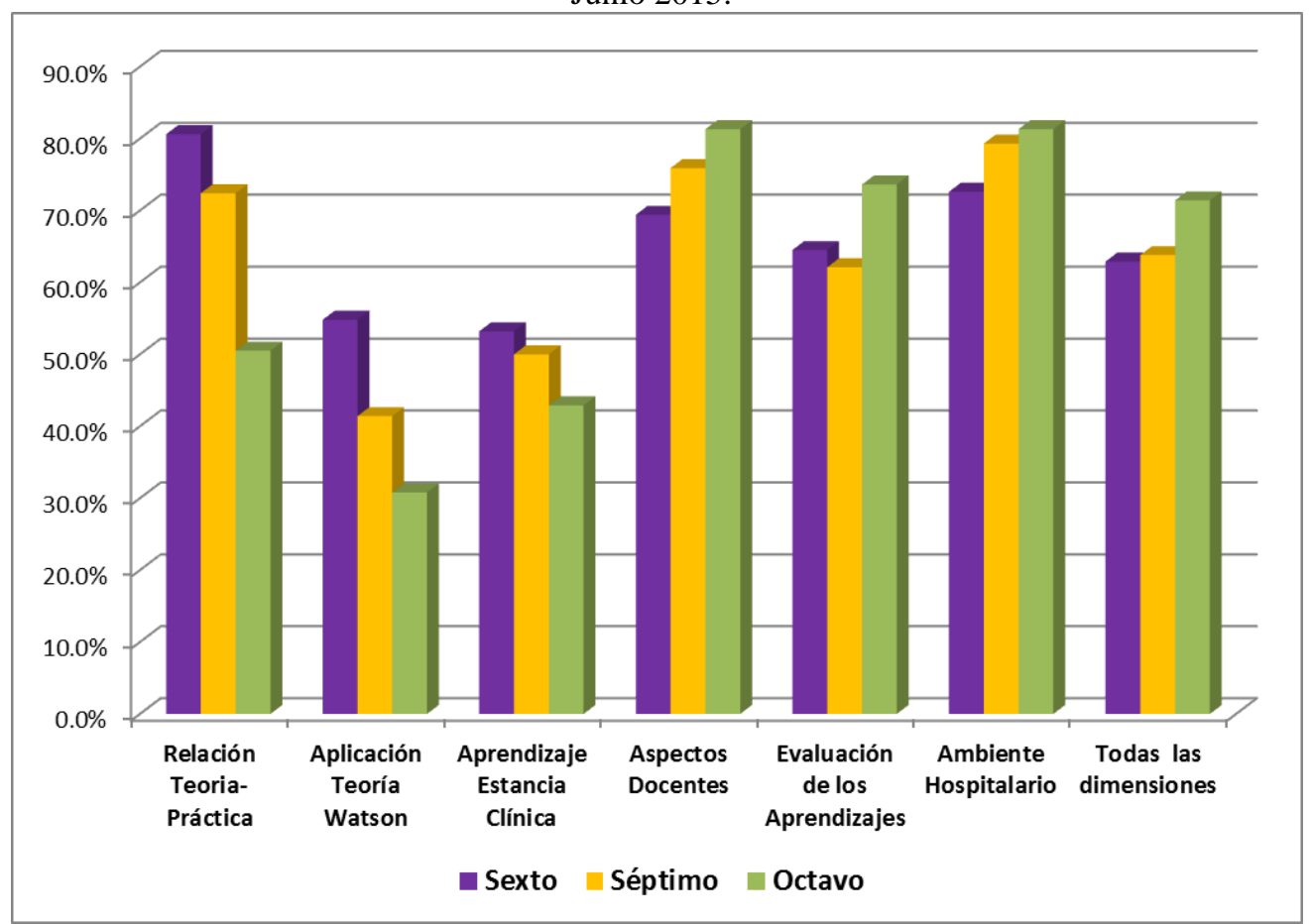

Fuente directa.

En la Figura 3 se muestran los resultados en la relación teoría y práctica, aplicación de la teoría de Jean Watson y aprendizaje en estancia clínica. Los porcentajes más elevados corresponden al sexto semestre, probablemente porque los estudiantes aún se encuentran anclados a una base más teórica que práctica.

En las dimensiones de competencia docente, evaluación del aprendizaje y ambiente hospitalario, los estudiantes del octavo semestre muestran porcentajes más favorables (70-80\%), esto debido a que han transitado por cuatro estancias clínicas y la teoría es cursada en solo 10 semanas. 
Los resultados coinciden con un estudio de Piña-Jiménez (2015), donde demuestra que los entornos de prácticas implican una actuación más activa en las tareas rutinarias de los hospitales y crean condiciones previas para la realización de procedimientos. Esta participación protagónica del estudiante en las prácticas clínicas también le permite observar el trato institucional a la persona de cuidado y encontrar -en algunos casos- la contradicción entre los valores que se enseñan en el contexto áulico y los escenarios reales de aplicación.

Figura 4. Percepción de los estudiantes en relación a la enseñanza-aprendizaje en la estancia clínica de la Licenciatura en Enfermería respecto al turno en el Ciclo Escolar Enero-Junio 2015.

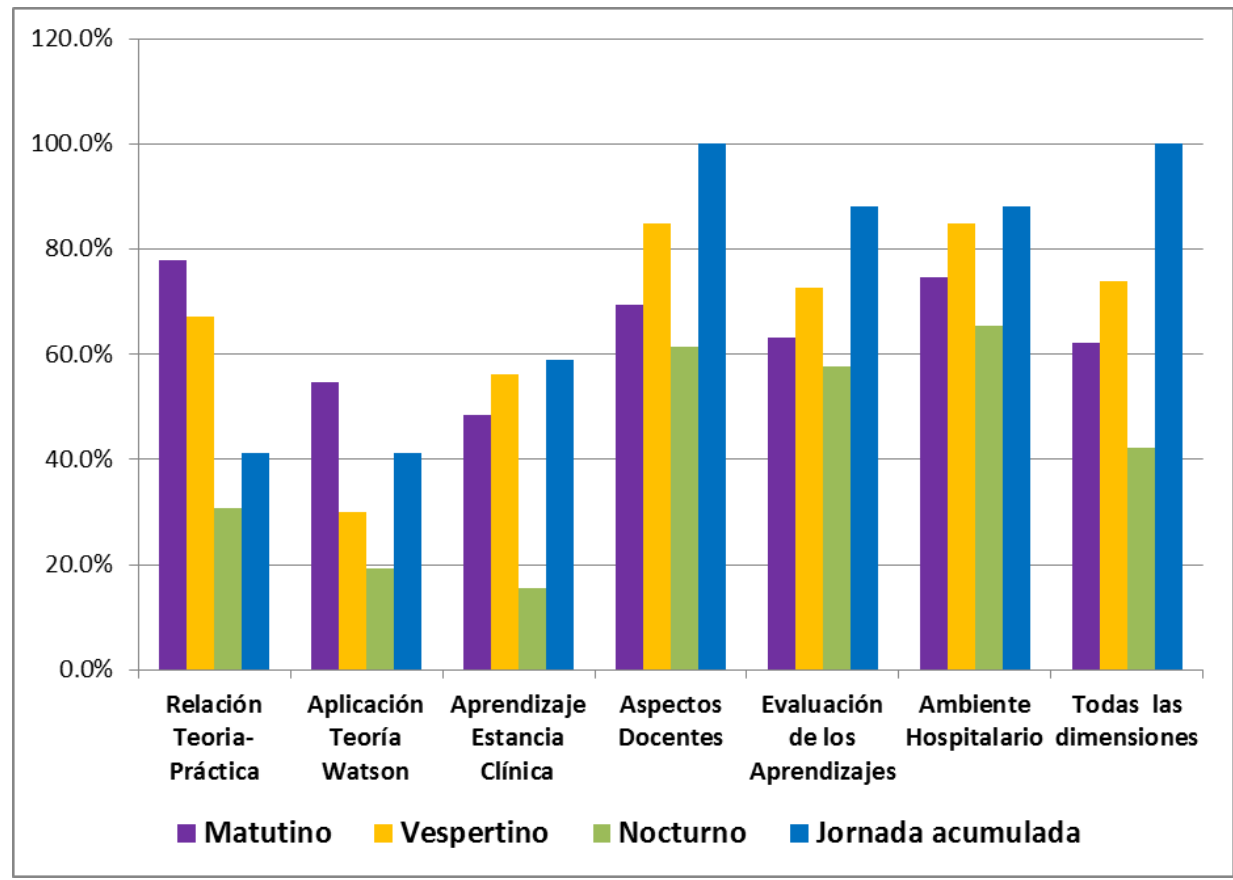

Fuente directa.

En la Figura 4 se aprecian los resultados más elevados en la relación teórico-práctica y aplicación de la teoría de Jean Watson. El turno matutino sobresale y el vespertino y nocturno muestra datos desfavorables.

En la dimensión de aprendizaje en estancia clínica, competencias del docente, evaluación del aprendizaje y ambiente hospitalario, el turno de jornada acumulada visualiza mayores porcentajes (58, 88 y $100 \%$ respectivamente). Es un dato que llama la atención ya que en fin de semana los recursos administrativos, humanos y materiales disminuyen, no así las personas de cuidado, elementos clave para lograr y evaluar el aprendizaje. Estos resultados orientan hacia una investigación complementaria sobre este turno para triangular la información. 
Figura 5. Percepción de los estudiantes de Licenciatura en Enfermería, respecto al contexto hospitalario para obtener aprendizaje en la estancia hospitalaria. Ciclo Escolar Enero - Junio 2015.

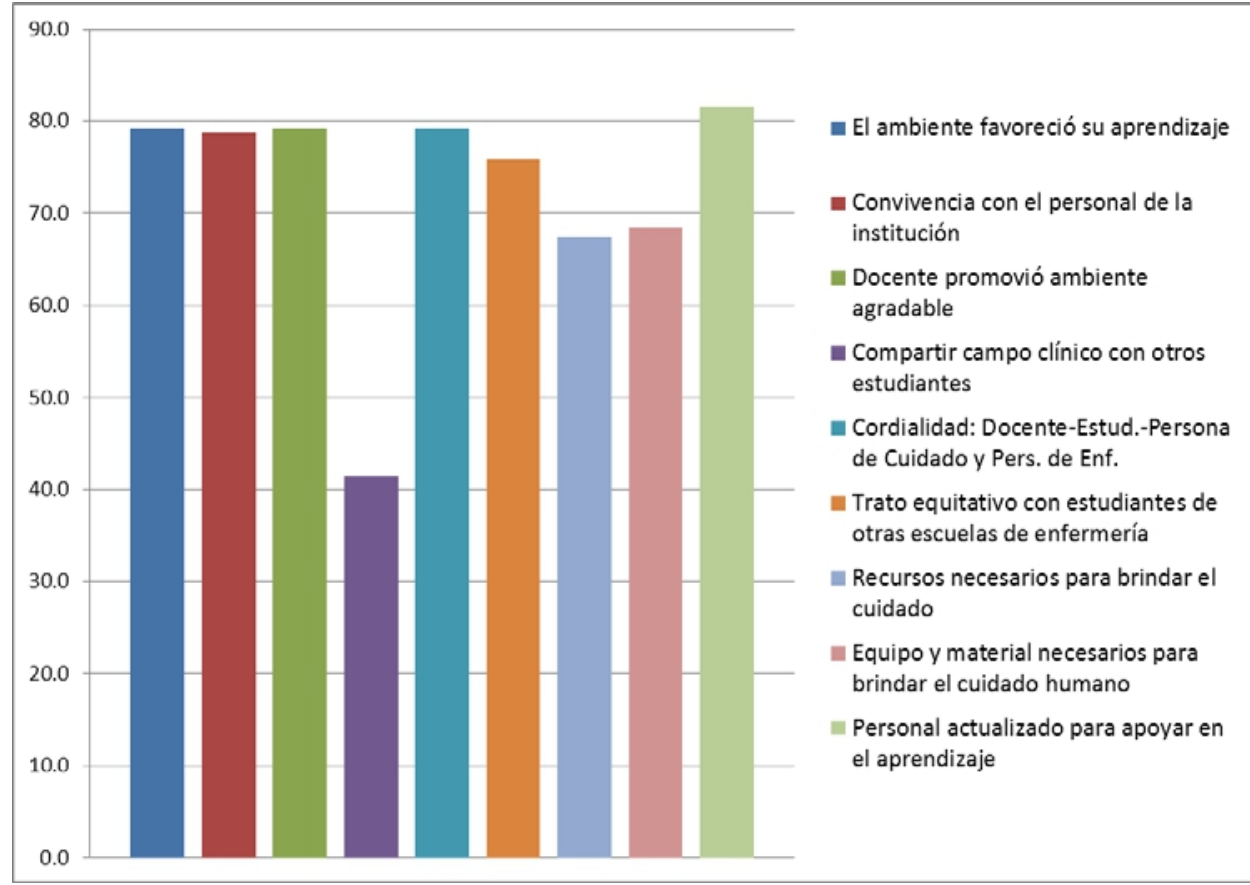

Fuente directa.

En la Figura 5 se visualiza la percepción de los estudiantes en relación al ambiente hospitalario, los porcentajes más elevados se muestran en el personal que se encuentra actualizado para apoyar el aprendizaje. En las dimensiones sobre la convivencia con el personal de la institución los resultados muestran que el ambiente favoreció el aprendizaje y el docente promovió un ambiente agradable.

Zarza Arizmendi M.D. y Luna Victoria F. M. (2014), en un estudio sobre los espacios o entornos del estudiante y el currículum del licenciado en enfermería de la Escuela Nacional de Enfermería y Obstetricia de la Universidad Nacional Autónoma de México, coinciden que la cotidianidad se conforma tanto por las actividades como por las experiencias del día a día en el aula, el laboratorio, y el hospital. Estas vivencias positivas constituyen su aprendizaje y el mejor instrumento para templar su personalidad en la dinámica de los centros o instituciones de salud.

\section{Conclusión}

La percepción es un proceso cognitivo que el ser humano enfrenta día a día, la formación disciplinar a nivel universitario no es la excepción ya que en el espacio áulico se generan los conocimientos y en los laboratorios la 
simulación y adquisición de habilidades. En este sentido el objetivo de este estudio es determinar la percepción que tiene el estudiante de enfermería sobre la enseñanza en la estancia clínica y si esta y si ésta se relaciona con la experiencia y el perfil docente para lograr el aprendizaje. La investigación se realizó en la Facultad de Enfermería y Nutriología de la Universidad Autónoma de Chihuahua en el periodo comprendido de marzo-diciembre del año 2015 en el cual de logró el objetivo mencionado. habilidades.

Además, toda línea curricular busca sensibilizar al estudiante sobre su entorno y desarrollar actitudes que impacten en su personalidad, proyectando valores como el respeto, la sensibilidad, el humanismo, entre otros., y que en cada uno de ellos le imprime conforme avanzan en su formación profesional.

Los estudiantes tienen experiencias favorables durante su proceso formativo que les permiten construir su identidad y una especie de empoderamiento disciplinar, el despertar de la conciencia acerca de su profesión y de las relaciones con la dinámica de mercado y el mundo global.

Sin embargo, existen muchas áreas de oportunidad en el ámbito hospitalario y comunitario, asuntos pendientes en el tema de la vinculación con las instituciones de salud y una exigencia en los entornos educativos para atender las observaciones y las necesidades de los estudiantes.

Durante su formación académica, el profesional de enfermería desarrolla competencias profesionales y una visión prospectiva para entender las necesidades del entorno, las exigencias de los escenarios reales de aplicación y una formación técnica, especializada y sensible para la praxis del cuidado.

En este sentido, es muy importante considerar la percepción de los estudiantes para redefinir las líneas institucionales y los modelos curriculares y así potenciar su proceso de enseñanza - aprendizaje durante los escenarios de práctica clínica y comunitaria.

Esta investigación, permitió discutir y analizar una serie de aspectos que tienen un impacto decisivo sobre la formación de los estudiantes de la licenciatura en enfermería. Reflexionar la práctica profesional, tomar decisiones que permitan potenciar el proceso de enseñanza-aprendizaje, promover una didáctica más funcional e ir acotando la brecha entre la teoría y la práctica, son algunos de los retos inmediatos que tienen los estudiantes y las instituciones en el área de la salud.

\section{References:}

1. Bernal Becerril, M. L. \& Ponce Gómez, G. (2009). Propuesta para la Enseñanza del cuidado en Enfermería. Rev. Enfermería Universitaria ENE-UNAM. Vol. 6 No. 1 Enero-Marzo 2009. Disponible en: http://www.medigrafhic.com/pdf/enfuni/eu-2009/eu091e.pdf 
2. Crespo-Knopfler, S. (2009). Percepción de los empleadores sobre el licenciado en enfermería de la Facultad de Estudios Superiores Zaragoza, Revista Enfermería Universitaria, ENEO-UNAM México, 6(1):8.

3. Crespo-Knopfler, S. González-Velázquez, MS. Agama Sarabia, A. (2011). Percepción de los alumnos de enfermería sobre los factores que inciden en su aprendizaje clínico. Vertientes Revista Especializada en Ciencias de la Salud, 14(2):88-93.

4. Mantilla-Pastrana, M. I., \& Gutiérrez-Agudelo, M. C. (2013). Procesos de evaluación del aprendizaje del cuidado en la práctica de estudiantes de Enfermería. Enfermería universitaria, 10(2), 43-49. [Recuperado en 26 de abril de 2016] Disponible en: http://www.scielo.org.mx/scielo.php?script=sci_arttext\&pid=S1665-

5. Moreno Tello, Mónica Aurora, Prado Moncivais, Elvia Nelly, García Avendaño, \& David Jahel. (2013). Percepción de los estudiantes de enfermería sobre el ambiente de aprendizaje durante sus prácticas clínicas. Revista CUIDARTE, [S.l.], v. 4, n. 1, dec. 2013. ISSN 2216-0973. Disponible en: $<$ https://www.revistacuidarte.org/index.php/cuidarte/article/view/3/1 16>. Fecha de acceso: 12 feb. 2017 doi:http://dx.doi.org/10.15649/cuidarte.v4i1.3

6. Palacios Gutiérrez Marcela \& Quiroga Lagos Pilar (2012). Percepción de los estudiantes de las características y comportamientos de sus profesores asociados a una enseñanza clínica efectiva. Estudios Pedagógicos, XXXVIII. Sin mes, 73-87. [Consultado: 27-04-2016] Disponible en: http://www.redalyc.org/comocitar.oa?id=173524158004

7. Pinto H. (2008). El Cuidado en el proceso de Enseñanza Aprendizaje. En: Grupo de Cuidado. El Arte y la Ciencia del Cuidado. Colombia: Universidad Nacional de Colombia; 2002.

8. Piña-Jiménez, I., \& Amador-Aguilar, R. (2015). La enseñanza de la enfermería con simuladores, consideraciones teórico-pedagógicas para perfilar un modelo didáctico. Enfermería universitaria, 12(3), 152-159. Disposnible https://dx.doi.org/10.1016/j.reu.2015.04.007

9. Pulido Mendoza, R. Aparicio Goñi, I. Mas Espejo, M. Tovar Reinoso, A. Rodríguez, \& García, M. (2008). Entorno del aprendizaje práctico-clínico. Percepción de los estudiantes de Enfermería. V Jornadas de Innovación Universitaria. Universidad Europea Madrid. 4-5 septiembre 2008. [Consultado: mayo 03, 2016]. Disponible en: http://universidadeuropea.es/myfiles/pageposts/jiu/jiu2008/archivos/S ALUD\%20DEDEPOR/Pulido,\%20Rosa.pdf 
10. Reyes, M.A., Mendoza, G., \& Barrera, P., (2015). Algunas Aportaciones psicológicas y Sociológicas a la Educación, Volumen II, 1ra edición, Ed. Nautilium A.C. México D.F.

11. Sanhueza Alvarado, O. \& Zavala, M. (2007). Enseñanza del cuidado humanizado en enfermería. Horizon enferm 2007; 18(2): 61-69. [Consultado 1-08-2016]. Disponible en: http://www.imbiomed.com

12. Uribe Romero Luis Rey \& Rivas Espinoza Juan Gabriel. (2010). Percepción de los Alumnos de Enfermería sobre el Desempeño docente en la Práctica Clínica. Rev. Enfermería Universitaria. ENEOUNAM. Vol. 7. Año 7. No. 4. (2010). [Consultado: 27-04-2016] Disponible en: http://www,revistas.unam.mx/index.php/reuindex

13. Zarza - Arizmendi M.D. \& Luna Victoria F.M. (2014). Espacios vividos de la práctica en el currículum del licenciado en enfermería de la Escuela Nacional de Enfermería y Obstetricia. Universidad Nacional Autónoma de México, México. Enfermería Universitaria 2014; 11(4): 132-138. 